\title{
Räumliche Effekte in elastischen Stößen von Kugeln
}

In diesem Kapitel werden zwei Aspekte genauer untersucht, deren Einflüsse bei der Vereinfachung der Bewegungsgleichungen zweier räumlich zusammenstoßender Kugeln in Abschn.2.2.1 als vernachlässigbar klein verworfen wurden. Dies betrifft einerseits einige dynamische Effekte durch die Rotation der Stoßachse (und deren Einfluss auf die Kontaktmechanik) und andererseits die torsionale Komponente des Stoßproblems.

\subsection{Einfluss der Rotation der Stoßachse}

In den bisherigen Kapiteln wurden dynamische Effekte in der räumlichen Kollision zweier Kugeln, die aus der (vergleichsweise langsamen) Rotation der Stoßachse resultieren, vernachlässigt. Unter anderem dadurch konnte das Stoßproblem auf den Stoß einer Kugel auf einen unendlich ausgedehnten Halbraum zurückgeführt werden (für den die Stoßachse offensichtlich fest ist). Schätzt man allerdings die einzelnen Beschleunigungsterme in den kinematischen Gl.(2.12) und (2.13) im Detail ab, ergibt sich ${ }^{1}$

$$
\begin{aligned}
& \dot{s}_{i} \approx \frac{s_{i, 0} v_{z, 0}}{d_{\max }}, \quad s_{i} \Omega \approx \frac{s_{i, 0} v_{x, 0}}{R_{1}+R_{2}}, \\
& \ddot{d} \approx \frac{v_{z, 0}^{2}}{d_{\max }}, \quad\left(R_{1}+R_{2}\right) \Omega^{2} \approx \frac{v_{x, 0}^{2}}{R_{1}+R_{2}}, \quad \dot{d} \Omega \approx \frac{v_{x, 0} v_{z, 0}}{R_{1}+R_{2}}, \quad\left(R_{1}+R_{2}\right) \dot{\Omega} \approx \frac{v_{x, 0} v_{z, 0}}{d_{\max }} .
\end{aligned}
$$

Hier bezeichnen $v_{z}$ und $v_{x}$ die normale und tangentiale relative Geschwindigkeit der kollidierenden Kugeln und $d_{\max }$ wie gewohnt die maximale Eindrucktiefe während des Stoßes. Der Index Null bezieht sich auf die Konfiguration zu Beginn der Kollision. Im Rahmen der Halbraumnäherung ist mit dem Kontaktradius $a$

\footnotetext{
${ }^{1}$ Da diese Beziehungen Abschätzungen darstellen, sind alle Größen betragsmäßig zu verstehen. 


$$
\frac{d_{\max }}{R_{1}+R_{2}} \approx\left(\frac{d_{\max }}{a_{\max }}\right)^{2}
$$

wobei das Verhältnis $d / a$ für die Gültigkeit der Halbraumhypothese klein sein muss. Der Stoßwinkel sei nun so flach, dass

$$
\frac{v_{z, 0}}{v_{x, 0}} \approx \frac{d_{\max }}{a_{\max }} .
$$

Bis auf den zentrifugalen Anteil $\left(R_{1}+R_{2}\right) \Omega^{2}$ sind dann immer noch alle langsamen dynamischen Effekte vernachlässigbar klein. Das Stoßproblem kann deswegen weiterhin auf den ebenen Stoß einer Kugel auf einen Halbraum zurückgeführt werden, wobei Gl. (2.34) allerdings durch

$$
\ddot{d}=\frac{F_{z}}{\tilde{m}}-\frac{v_{x}^{2}}{R_{1}+R_{2}}-\left(R_{1}+R_{2}\right) \Omega_{x}^{2}
$$

ersetzt werden muss. Dabei ist $\Omega_{x}$ eine Konstante, da weiterhin angenommen sei, dass die Kontaktkraft in der Stoßebene liegt, die entsprechend nicht verlassen wird. Die Gl. (2.33) und (2.35) bleiben unverändert gültig. Der zentrifugale Anteil der Beschleunigung ${ }^{2}$ sorgt dabei offensichtlich für eine zusätzliche Abstoßung (neben der Normalkraft im Kontakt) der Kugeln während der Kollision. Der Einfluss dieser zusätzlichen Abstoßung auf das Stoßproblem soll im Folgenden für den reibungsfreien Stoß mit und ohne Adhäsion und den Stoß mit Reibung genauer untersucht werden.

\subsubsection{Reibungsfreier Stoß ohne Adhäsion}

Im Fall des reibungsfreien Stoßes verschwindet die tangentiale Komponente der Kontaktkraft und $v_{x}$ ist während der Kollision konstant. Mit der Lösung des Hertzschen Kontaktproblems erhält man so

$$
\tilde{m}\left(\ddot{d}+a_{0}\right)=-\frac{4}{3} \tilde{E} \sqrt{\tilde{R} d^{3}}, \quad a_{0}:=\frac{v_{x, 0}^{2}}{R_{1}+R_{2}}+\left(R_{1}+R_{2}\right) \Omega_{x}^{2}
$$

als Bewegungsgleichung mit den Anfangsbedingungen

$$
\dot{d}(t=0)=\left|v_{z, 0}\right|, \quad d(t=0)=0
$$

\footnotetext{
${ }^{2}$ Im ebenen Modell ist das natürlich keine zentrifugale Kraft, sondern einfach eine zusätzliche Kraft in Normalenrichtung; trotzdem soll im Folgenden die Bezeichnung ,zentrifugal“" beibehalten werden, da dies der physikalische Ursprung der betrachteten Effekte bei der räumlichen Kollision ist.
} 
für die Indentierungstiefe $d$. In Gl. (7.6) wurden außerdem die aus den Gl. (2.17), (3.14) und (2.28) bekannten Definitionen der effektiven Werte der Masse, des Elastizitätsmoduls und des Krümmungsradius im Kontakt verwendet.

Führt man die dimensionsfreien Größen

$$
\hat{t}:=\frac{\left|v_{z, 0}\right|}{d_{0}} t=\frac{t}{t_{0}}, \quad \hat{d}:=\frac{d}{d_{0}},
$$

ein - wobei $d_{0}$ die maximale Eindrucktiefe im Fall ohne zentrifugalen Beitrag bezeichnet, die Gl.(5.8) entnommen werden kann - erhält man die normierte Bewegungsgleichung

$$
\frac{\mathrm{d}^{2} \hat{d}}{\mathrm{~d} \hat{t}^{2}}+\frac{5}{4} \hat{d}^{3 / 2}+\alpha_{0}=0, \quad \alpha_{0}:=\frac{a_{0} d_{0}}{v_{z, 0}^{2}}
$$

mit den Anfangsbedingungen

$$
\frac{\mathrm{d} \hat{d}}{\mathrm{~d} \hat{t}}(\hat{t}=0)=1, \quad \hat{d}(\hat{t}=0)=0 .
$$

Integration über die (normierte) Eindrucktiefe liefert die Energiebilanz

$$
\left(\frac{\mathrm{d} \hat{d}}{\mathrm{~d} \hat{t}}\right)^{2}=1-\hat{d}^{5 / 2}-2 \alpha_{0} \hat{d}
$$

Die maximale Eindrucktiefe ergibt sich daher als Lösung der Gleichung

$$
0=1-\hat{d}_{\max }^{5 / 2}-2 \alpha_{0} \hat{d}_{\max }
$$

Im Gegensatz zum idealen Hertzschen Stoßproblem (mit $\alpha_{0}=0$ ) ist Gl.(7.11) nicht geschlossen analytisch lösbar. Eine numerische Lösung ist für verschiedene Werte von $\alpha_{0}$ in Abb.7.1 gezeigt. Offenbar werden durch den Einfluss der Zentrifugalkraft die maximale Eindrucktiefe und die Stoßdauer kleiner. Die Form der Lösung - und insbesondere die Symmetrie zwischen Kompressions- und Restitutionsphase - bleiben aber unverändert. Außerdem ist die Stoßzahl aus diesem Grund weiterhin Eins.

In der Regel wird die normierte zentrifugale Beschleunigung $\alpha_{0}$ klein sein. In diesem Fall können für die Gl. (7.11) und (7.12) asymptotische Näherungslösungen in geschlossen analytischer Form gefunden werden. Die asymptotische Lösung von Gl.(7.12) ist

$$
\hat{d}_{\max }=1-\frac{4}{5} \alpha_{0}+\mathcal{O}\left(\alpha_{0}^{2}\right) .
$$

Die Lösung der Bewegungsgleichung kann man in der Reihe

$$
\hat{d}(\hat{t})=\sum_{i=0}^{\infty} \delta_{i}(\hat{t}) \alpha_{0}^{i}
$$


Abb.7.1 Normierte Trajektorien während des räumlichen Normalstoßes elastischer Kugeln für verschiedene normierte Zentrifugalbeschleunigungen $\alpha_{0}$

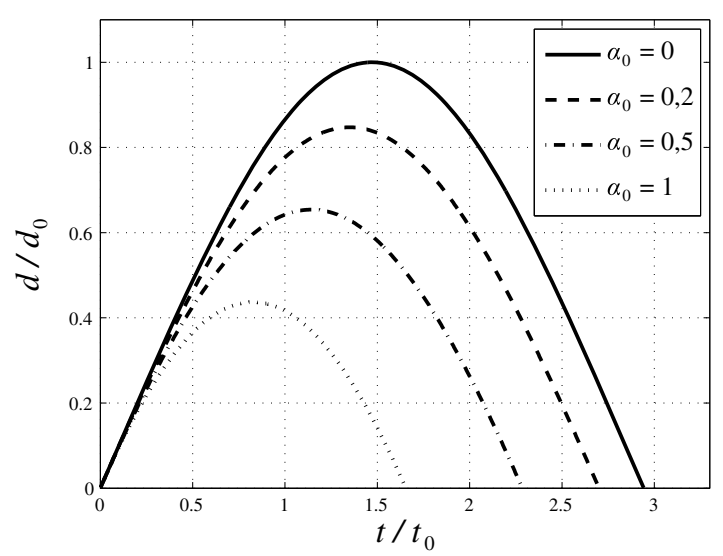

entwickeln. Für die Beiträge nullter Ordnung ergibt sich aus Gl.(7.11) die Beziehung

$$
\left(\frac{\mathrm{d} \delta_{0}}{\mathrm{~d} \hat{t}}\right)^{2}=1-\delta_{0}^{5 / 2}
$$

Dies ist die normierte Energiebilanz des idealen Hertzschen Stoßproblems mit der in Gl. (5.11) gegebenen (impliziten) Lösung

$$
\hat{t}=\frac{2}{5} \mathrm{~B}\left(\delta_{0}^{5 / 2} ; \frac{2}{5}, \frac{1}{2}\right),
$$

wobei B die im Anhang beschriebene unvollständige Beta-Funktion bezeichnet. Die Terme der Ordnung $\alpha_{0}$ in Gl.(7.11) liefern die Beziehung

$$
\frac{\mathrm{d} \delta_{0}}{\mathrm{~d} \hat{t}} \frac{\mathrm{d} \delta_{1}}{\mathrm{~d} \hat{t}}=-\frac{5}{4} \delta_{0}^{3 / 2} \delta_{1}-\delta_{0}
$$

die sich mithilfe von Gl.(7.15) in das gewöhnliche lineare Randwertproblem

$$
\left(1-\delta_{0}^{5 / 2}\right) \frac{\mathrm{d} \delta_{1}}{\mathrm{~d} \delta_{0}}=-\frac{5}{4} \delta_{0}^{3 / 2} \delta_{1}-\delta_{0}, \quad \delta_{1}\left(\delta_{0}=0\right)=0
$$

mit der Lösung

$$
\delta_{1}\left(\delta_{0}\right)=-\frac{4}{5} \delta_{0}^{2}+\frac{6}{25} \sqrt{1-\delta_{0}^{5 / 2}} \mathrm{~B}\left(\delta_{0}^{5 / 2} ; \frac{4}{5}, \frac{1}{2}\right)
$$

überführen lässt. Abb. 7.2 zeigt einen Vergleich der vollständigen numerischen Lösung mit der oben bestimmten analytischen Asymptote bis zur ersten Ordnung für $\alpha_{0}=0,2$. Offenbar stimmt die asymptotische Näherung sehr gut mit der ,exakten“ numerischen Lösung überein. 
Abb. 7.2 Vergleich der vollständigen numerischen Lösung für die normierte Trajektorie (Kreise) mit der analytischen asymptotischen Näherung bis zur ersten Ordnung (durchgezogene Linie) für den Zusammenstoß homogener Kugeln mit $\alpha_{0}=0,2$

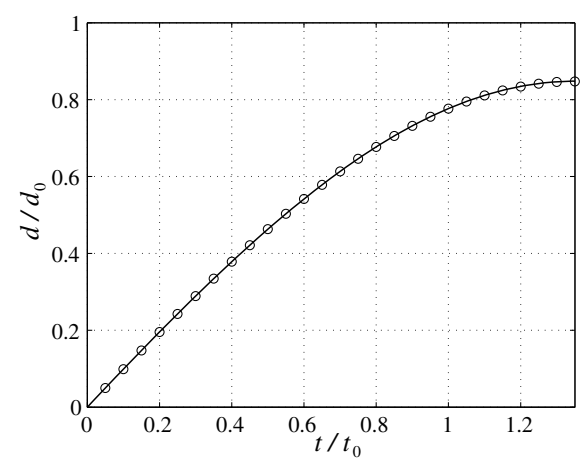

\section{Funktionale Gradientenmedien}

Die oben erhaltenen Ergebnisse können ohne Schwierigkeiten für elastische Kugeln verallgemeinert werden, bei denen der elastische Modul mit der Tiefe entsprechend eines Potenzgesetzes mit dem Exponenten $k$ variiert - siehe Gl.(3.209). Die normierte Energiebilanz nimmt die Form

$$
\left(\frac{\mathrm{d} \hat{d}}{\mathrm{~d} \hat{t}}\right)^{2}=1-\hat{d}^{\frac{5+k}{2}}-2 \alpha_{0} \hat{d}
$$

an, wobei die Normierungstiefe $d_{0}$ Gl. (5.21) entnommen werden kann. Die maximale Indentierungstiefe während des Stoßes beträgt in erster Näherung

$$
\hat{d}_{\max }=1-\frac{4}{5+k} \alpha_{0}+\mathcal{O}\left(\alpha_{0}^{2}\right)
$$

Die nullte Ordnung der asymptotischen Lösung der Bewegungsgleichung ist durch die in Gl.(5.22) gegebene Lösung ohne zentrifugale Beiträge bestimmt,

$$
\hat{t}=\frac{2}{5+k} \mathrm{~B}\left(\delta_{0}^{\frac{5+k}{2}} ; \frac{2}{5+k}, \frac{1}{2}\right) .
$$

Für die erste Ordnung erhält man durch eine den obigen Überlegungen völlig analoge Rechnung

$$
\delta_{1}\left(\delta_{0}\right)=-\frac{4}{5+k} \delta_{0}^{2}+\frac{2(3-k)}{(5+k)^{2}} \sqrt{1-\delta_{0}^{\frac{5+k}{2}}} \mathrm{~B}\left(\delta_{0}^{\frac{5+k}{2}} ; \frac{4}{5+k}, \frac{1}{2}\right) .
$$

Für $k=0$ ergeben sich natürlich die bereits gezeigten homogenen Ergebnisse. 


\subsubsection{Reibungsfreier Stoß mit JKR-Adhäsion}

Die zentrifugale Beschleunigung durch die Rotation der Stoßachse hat ebenfalls einen Einfluss auf das adhäsive Normalstoßproblem, insbesondere darauf, ob die kollidierenden Kugeln durch die Wirkung der Adhäsion aneinander kleben bleiben oder tatsächlich voneinander zurückprallen.

Dabei muss man sich zunächst vergegenwärtigen, dass die initiale und die kritische Kontaktkonfiguration zu Beginn und am Ende des Stoßes nur durch die Kontaktwechselwirkung bestimmt und unabhängig von zentrifugalen Einflüssen sind. Die Änderung der kinetischen Energie während des Stoßes ist daher elementar durch

$$
\Delta U_{\mathrm{kin}}=\Delta U_{\mathrm{kin}, 0}-\tilde{m} a_{0} d_{c}^{\mathrm{WS}}
$$

gegeben. Hier bezeichnet $\Delta U_{\text {kin, } 0}$ die Energieänderung durch die adhäsive Wechselwirkung, die in Gl.(5.36) nachgeschlagen werden kann, $d_{c}^{\mathrm{WS}}$ die kritische Indentierungstiefe, bei der der JKR-adhäsive Normalkontakt unter weggesteuerten Bedingungen seine Stabilität verliert - siehe Gl.(3.60) - und $a_{0}$ die in Gl. (7.6) definierte zentrifugale Beschleunigung. Aus der Beziehung (7.24) folgt, dass die Kugeln aneinander kleben bleiben, falls

$$
\left(\frac{v_{z, 0}}{v_{1}}\right)^{2}+\left(\frac{v_{x, 0}}{v_{2}}\right)^{2} \leq 1,
$$

wobei

$$
v_{1}^{2}:=\frac{2 \Delta U_{\mathrm{kin}, 0}}{\tilde{m}}, \quad v_{2}^{2}:=\frac{v_{1}^{2}}{2} \frac{R_{1}+R_{2}}{\left|d_{c}^{\mathrm{WS}}\right|} .
$$

Gl. (7.25) beschreibt offensichtlich das Innere einer Ellipse mit den Halbachsen $v_{1}$ und $v_{2}$. Durch die zusätzliche Abstoßung aus der Rotation der Stoßachse können sich also auch kollidierende Kugeln voneinander lösen, deren relative Normalgeschwindigkeit in einem reinen adhäsiven Normalstoß dafür eigentlich zu klein wäre.

\subsubsection{Stoß mit Reibung ohne Adhäsion}

Was ändert sich nun durch das Wechselspiel zwischen Reibung und dem zentrifugalen Anteil der radialen Beschleunigung? Um die Anzahl variierbarer Parameter klein zu halten, soll im Folgenden der Beitrag durch $\Omega_{x}$ in Gl. (7.5) vernachlässigt werden. Die Charakteristika der Lösung entstehen durch den Beitrag aus $v_{x}$, oder besser gesagt daraus, dass sich $v_{x}$ durch die Reibung während des Stoßes verändert (dies gilt für $\Omega_{x}$, wie beschrieben, nicht). Da außerdem der Stoßwinkel sehr flach sein muss, damit die zentrifugalen Beiträge relevant werden, wird der Kontakt für realistische Parameterkombinationen während der ganzen Kollision vollständig gleiten. Dadurch wird die Kontaktmechanik des Problems trivial und die Form 
der Bewegungsgleichungen sehr einfach. Man erhält mithilfe der Gl. (7.5) und (2.33) sowie dem Amontons-Coulomb-Gesetz und der Hertzschen Lösung des Normalkontaktproblems ${ }^{3}$

$$
\begin{aligned}
\tilde{m}\left(\ddot{d}+\frac{v_{x}^{2}}{R_{1}+R_{2}}\right) & =-\frac{4}{3} \tilde{E} \sqrt{\tilde{R} d^{3}} \\
\tilde{m} \dot{v}_{x} & =-\frac{4}{3} \mu \tilde{E} \sqrt{\tilde{R} d^{3}}
\end{aligned}
$$

Mit den dimensionsfreien Größen

$$
\hat{t}:=\frac{\left|v_{z, 0}\right|}{d_{0}} t, \quad \hat{d}:=\frac{d}{d_{0}}, \quad \hat{v}_{x}:=\frac{v_{x}}{\left|v_{x, 0}\right|}
$$

lässt sich das auf die Form

$$
\begin{array}{rlrl}
\frac{\mathrm{d}^{2} \hat{d}}{\mathrm{~d} \hat{t}^{2}}+\frac{5}{4} \hat{d}^{3 / 2}+\delta \hat{v}_{x}^{2} & =0, & \delta: & :=\frac{v_{x, 0}^{2}}{v_{z, 0}^{2}} \frac{d_{0}}{R_{1}+R_{2}}, \\
\frac{\mathrm{d} \hat{v}_{x}}{\mathrm{~d} \hat{t}}+\frac{5}{4} \beta \hat{d}^{3 / 2} & =0, \quad \beta:=\frac{\mu\left|v_{z, 0}\right|}{\left|v_{x, 0}\right|},
\end{array}
$$

mit den Anfangsbedingungen

$$
\hat{v}_{x}(\hat{t}=0)=1, \quad \hat{v}_{z}(\hat{t}=0)=1, \quad \hat{d}(\hat{t}=0)=0,
$$

bringen. Dabei sind $v_{z, 0}$ und $v_{x, 0}$ die normale und tangentiale Komponente der Geschwindigkeit des Schwerpunkts vor dem Stoß und $d_{0}$ die maximale Eindrucktiefe im Hertzschen Stoßproblem ohne Berücksichtigung zentrifugaler Beiträge, die in Gl. (5.8) nachgeschlagen werden kann. Offensichtlich hängt die Lösung des Stoßproblems in normierten Größen nur von den beiden Parametern $\delta$ und $\beta$ ab.

Die sich ergebende kinematische Stoßzahl für die normale Bewegung des Schwerpunkts, $\epsilon_{z}$, ist in Abb. 7.3 als Konturliniendiagramm in Abhängigkeit der bestimmenden Parameter gezeigt. Man erkennt deutlich, dass die normale Stoßzahl in Fällen mit Zentrifugalterm und Reibung kleiner ist als Eins; das liegt daran, dass die tangentiale Geschwindigkeit $v_{x}$ durch die Reibung während der Kollision kleiner wird; die Verläufe der gesamten Kraft in vertikaler Richtung (bestehend aus der Normalkraft im Kontakt und dem zentrifugalen Beitrag) während der Kompressions- und Restitutionsphase sind deswegen nicht symmetrisch und die Stoßzahl daher kleiner als Eins.

\footnotetext{
${ }^{3}$ Da der zentrifugale Anteil durch die Tangentialbewegung des Schwerpunktes bestimmt wird, ist es sinnvoller, die tangentiale Bewegungsgleichung bezüglich des Schwerpunktes aufzustellen, anstatt, wie im vorherigen Kapitel, bezüglich des Kontaktpunktes.
} 
Abb.7.3 Kinematische Stoßzahl der Normalbewegung des Schwerpunkts der Kugel $\epsilon_{z}$ für den Stoß mit Reibung unter Berücksichtigung zentrifugaler Beschleunigungsanteile. Konturliniendiagramm in Abhängigkeit der beiden bestimmenden Parameter $\delta$ und $\beta$

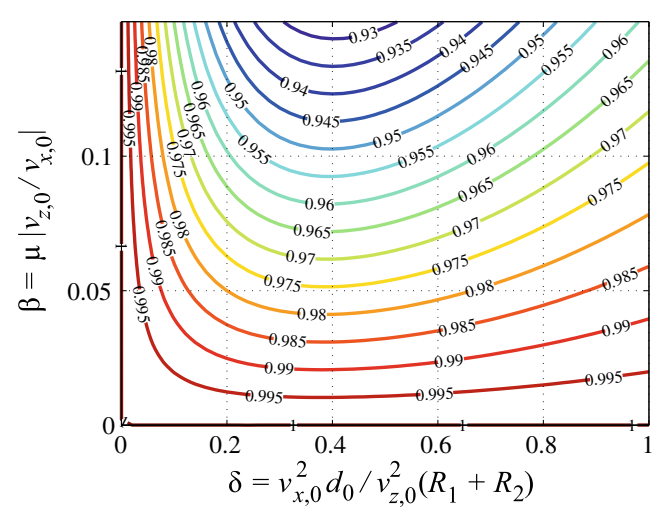

\subsection{Elastischer Torsionsstoß}

Im folgenden Unterkapitel wird das torsionale Stoßproblem von elastischen Kugeln genauer untersucht. Dabei sollen, wie in den vorherigen Kapiteln, alle Prozesse quasistatisch ablaufen, um von den statischen Kontaktlösungen aus dem dritten Kapitel Gebrauch machen zu können. Im zweiten Kapitel wurde begründet, dass die torsionale Komponente des Stoßproblems im Rahmen der Gültigkeit der Halbraumnäherung sehr klein ist. Das liegt daran, dass die charakteristische Frequenz der torsionalen Bewegung sehr viel kleiner als die der Indentierung ist, die relative axiale Winkelgeschwindigkeit $\omega_{z}$ ändert sich also nur sehr langsam. Trotzdem kann diese Änderung und die damit assoziierte Stoßzahl

$$
\epsilon_{t}:=-\frac{\omega_{z}\left(t=T_{S}\right)}{\omega_{z, 0}}
$$

von Interesse sein.

Im Fall des reinen Torsionsproblems (Normalbewegung und reine Rotation um die Normalenachse) könnte man den Stoß auch für beliebige axialsymmetrische Körper untersuchen ${ }^{4}$, da sich dann die Orientierung der Körper im Raum während des Stoßes nicht ändert und die makroskopische Dynamik daher elementar ist. Die Kontaktmechanik des Torsionsproblems ist aber in anderen Fällen als dem parabolischen Kontakt mathematisch sehr unhandlich (obwohl physikalisch-konzeptuell ohne Schwierigkeiten möglich), deswegen steht im Folgenden nur der Kontakt von Kugeln im Fokus der Untersuchung.

Der Drallsatz um die Normalen-, bzw. Symmetrieachse

$$
M_{z}=-J^{*} \dot{\omega}_{z}, \quad J^{*}:=\frac{J_{1}^{S} J_{2}^{S}}{J_{1}^{S}+J_{2}^{S}},
$$

\footnotetext{
${ }^{4}$ Es sei dabei angenommen, dass die Normalenachse mit der Symmetrieachse der Körper zusammenfällt.
} 
mit den Trägheitsmomenten der beiden Kugeln $J_{i}^{S}$ um die Normalenachse, liefert bei Kenntnis des Torsionsmomentes $M_{z}$ die Bewegungsgleichung für die (relative) torsionale Rotation. Die Aufgabe besteht nun in der kontaktmechanischen Bestimmung des Moments und der Integration der entstehenden Bewegungsgleichung.

Die vollständige Lösung des elastischen Torsionsstoßproblems von Kugeln mit einem unendlichen oder endlichen Reibbeiwert stammt von Jäger [1]. Es soll zunächst der Fall ohne lokales Gleiten im Kontakt betrachtet und anschließend der Einfluss des lokalen Gleitens untersucht werden.

\subsubsection{Stoß ohne Gleiten}

Die Mechanik des torsionalen Stoßes von elastischen Kugeln ohne Gleiten ist qualitativ sehr ähnlich zu dem entsprechenden allgemeinen ebenen Stoßproblem: Die Kompressionsund Restitutionsphase müssen getrennt voneinander untersucht werden, da beide qualitativ unterschiedliches Verhalten zeigen. In der Kompressionsphase kann man die Bewegungsgleichung inkrementell schreiben; in der Restitutionphase formuliert man eine explizite Bewegungsgleichung für die Differenz zwischen zwei Kontaktkonfigurationen mit dem gleichen Kontaktradius während der Kompressions- und Restitutionsphase.

\section{Kompressionsphase}

Wenn die Indentierungstiefe und damit der Kontaktradius monoton wachsen, kann der inkrementelle Beitrag zu dem Torsionsmoment durch eine Starrkörperrotation des Kontaktgebiets mit dem momentanen Radius $a$ um den differentiellen Winkel $\omega_{z} \mathrm{~d} t$ bestimmt werden. Mit Gl. (3.131) und dem Drallsatz (7.34) lautet dann die Bewegungsgleichung wie folgt:

$$
\ddot{\omega}_{z}+\frac{16 G}{3 J^{*}} a^{3} \omega_{z}=0 .
$$

Dabei bezeichnet $G$ den in Gl.(3.12) definierten Schubmodul. Verwendet man die dimensionsfreien Größen

$$
\hat{\omega}_{z}:=\frac{d_{\max }}{\left|v_{z, 0}\right|} \omega_{z}, \quad \hat{t}:=\frac{\left|v_{z, 0}\right|}{d_{\max }} t, \quad \xi:=\left(\frac{d}{d_{\max }}\right)^{5 / 2}, \quad \chi_{t}:=(1-v) \frac{\tilde{m} \tilde{R}^{2}}{J^{*}} \frac{d_{\max }}{\tilde{R}},
$$

mit der Stoßgeschwindigkeit $v_{z, 0}$, der maximalen Indentierungstiefe $d_{\max }$ aus Gl. (5.8), sowie den effektiven Werten der Masse und des Krümmungsradius, $\tilde{m}$ und $\tilde{R}$, lautet die Bewegungsgleichung in normierter Form

$$
\frac{\mathrm{d}^{2} \hat{\omega}_{z}}{\mathrm{~d} \hat{t}^{2}}+\frac{5}{2} \chi_{t} \xi^{3 / 5} \hat{\omega}_{z}=0
$$

mit den Anfangsbedingungen

$$
\hat{\omega}_{z}(\hat{t}=0)=\hat{\omega}_{z, 0}, \quad \frac{\mathrm{d} \hat{\omega}_{z}}{\mathrm{~d} \hat{t}}(\hat{t}=0)=0 .
$$


Außerdem gilt natürlich die aus der Lösung des Normalstoßproblems bekannte (und bereits für das ebene Problem verwendete) Beziehung

$$
\frac{\mathrm{d} \xi}{\mathrm{d} \hat{t}}=\frac{5}{2} \xi^{3 / 5}(1-\xi)^{1 / 2}
$$

Gl. (7.37) kann leider, trotz der ins Auge springenden Ähnlichkeit, nicht analog zu Gl. (6.5) für die Tangentialbewegung des Kontaktpunktes im ebenen Stoß ohne Gleiten gelöst werden. Allerdings muss $\chi_{t}$ - dies ist das Analogon für das Torsionsproblem zu dem aus dem ebenen Problem bekannten Parameter $\chi$ - im Rahmen der Halbraumnäherung offensichtlich klein sein. Es ist daher vorteilhaft, die Lösung in einer Potenzreihe

$$
\hat{\omega}_{z}(\hat{t})=\sum_{i=0}^{\infty} \chi_{t}^{i} \hat{\omega}_{z}^{(i)}(\hat{t})
$$

zu entwickeln. Das nullte Glied ist dabei offensichtlich

$$
\hat{\omega}_{z}^{(0)}(\hat{t}) \equiv \hat{\omega}_{z, 0}
$$

Für das erste Störglied erhält man deswegen das Anfangswertproblem

$$
\frac{\mathrm{d}^{2} \hat{\omega}_{z}^{(1)}}{\mathrm{d} \hat{t}^{2}}+\frac{5}{2} \chi_{t} \xi^{3 / 5} \hat{\omega}_{z, 0}=0, \quad \hat{\omega}_{z}^{(1)}(\hat{t}=0)=0, \quad \frac{\mathrm{d} \hat{\omega}_{z}^{(1)}}{\mathrm{d} \hat{t}}(\hat{t}=0)=0
$$

das mit Gl. (7.39) zu

$$
\hat{\omega}_{z}^{(1)}(\hat{t})=2 \hat{\omega}_{z, 0}\left(\xi^{2 / 5}-\hat{t}\right)
$$

aufgelöst werden kann. Am Umkehrpunkt der Normalbewegung ergibt sich damit die normierte Winkelgeschwindigkeit - die höheren Störglieder werden vernachlässigt, da $\chi_{t}$ klein sein muss -

$$
\hat{\omega}_{z, m} \approx \hat{\omega}_{z, 0}\left[1-\chi_{t}\left(\hat{T}_{S}-2\right)\right]
$$

mit der normierten Stoßdauer - siehe Gl.(5.12) -

$$
\hat{T}_{S}:=\frac{4}{5} \mathrm{~B}\left(1 ; \frac{2}{5}, \frac{1}{2}\right) .
$$

\section{Restitutionsphase}

Das Verhalten in der Restitutionsphase gehorcht den gleichen qualitativen Mechanismen wie im Fall des ebenen Stoßes ohne Gleiten. Während der Restitutionsphase gibt es für jeden Zeitpunkt $t>T_{S} / 2$ einen Zeitpunkt $t_{c}(t)=T_{S}-t$ während der Kompressionsphase, sodass $a(t)=a\left(t_{c}\right)$. Die Differenz der Kontaktmomente zwischen diesen beiden Zeitpunkten ergibt sich aus der in dieser Zeit erfolgten Starrkörperrotation. Mit Gl.(3.131) erhält man daher 


$$
M_{z}(t)-M_{z}\left(t_{c}\right)=\frac{16 G}{3} a^{3}\left(\varphi_{z}(t)-\varphi_{z}\left(t_{c}\right)\right) .
$$

Da der Drallsatz (7.34) für alle Zeitpunkte Gültigkeit hat, erhält man für die Differenz der Verdrehwinkel

$$
\varphi_{z}^{*}(t):=\varphi_{z}(t)-\varphi_{z}\left(t_{c}\right)
$$

die normierte Bewegungsgleichung

$$
\frac{\mathrm{d}^{2} \varphi_{z}^{*}}{\mathrm{~d} \hat{t}^{2}}+\frac{5}{2} \chi_{t} \xi^{3 / 5} \varphi_{z}^{*}=0
$$

mit den sich aus der Symmetrie ergebenden Anfangsbedingungen

$$
\varphi_{z}^{*}\left(\hat{t}=\frac{\hat{T}_{S}}{2}\right)=0, \quad \frac{\mathrm{d} \varphi_{z}^{*}}{\mathrm{~d} \hat{t}}\left(\hat{t}=\frac{\hat{T}_{S}}{2}\right)=2 \hat{\omega}_{z, m} .
$$

Außerdem muss in Gl. (7.39) für die Restitution das Vorzeichen gewechselt werden.

Entwickelt man $\varphi_{z}^{*}$ analog zur Kompressionsphase in einer Potenzreihe in $\chi_{t}$, ergibt sich für die ersten beiden Glieder

$$
\begin{aligned}
\varphi_{z}^{*(0)} & =2 \hat{\omega}_{z, m}\left(\hat{t}-\frac{\hat{T}_{S}}{2}\right), \\
\frac{\mathrm{d} \varphi_{z}^{*(1)}}{\mathrm{d} \hat{t}} & =-4 \hat{\omega}_{z, m}\left[\left(\hat{t}-\frac{\hat{T}_{S}}{2}\right) \sqrt{1-\xi}+\xi^{2 / 5}-1\right] .
\end{aligned}
$$

Vernachlässigt man wiederum alle höheren Störglieder, ist die normierte Winkelgeschwindigkeit am Ende des Stoßes

$$
\hat{\omega}_{z}\left(\hat{t}=\hat{T}_{S}\right) \approx 2 \hat{\omega}_{z, m}\left[1-\chi_{t}\left(\hat{T}_{S}-2\right)\right]-\hat{\omega}_{z, 0}=-\hat{\omega}_{z, 0}\left\{1-2\left[1-\chi_{t}\left(\hat{T}_{S}-2\right)\right]^{2}\right\}
$$

und damit die torsionale Stoßzahl

$$
\begin{aligned}
\epsilon_{t} & \approx 1-2\left[1-\chi_{t}\left(\hat{T}_{S}-2\right)\right]^{2} \\
& \approx-1+4 \chi_{t}\left(\hat{T}_{S}-2\right) .
\end{aligned}
$$

Diese analytischen Näherungen sind in Abb. 7.4 gemeinsam mit einer vollständigen numerischen Lösung gezeigt. Offenbar stimmt die lineare Näherung (7.54) bis etwa $\chi_{t}=0,05$ sehr gut mit der korrekten Lösung überein, die quadratische Näherung (7.53) sogar bis etwa $\chi_{t}=0,1$. 
Abb. 7.4 Torsionale Stoßzahl $\epsilon_{t}$ als Funktion des Parameter $\chi_{t}$. Die Kreise bezeichnen eine vollständige numerische Lösung auf Grundlage der Bewegungsgleichungen (7.37) und (7.48), die Linien beschreiben die analytischen Näherungen (7.53) (gestrichelt) und (7.54) (durchgezogen)

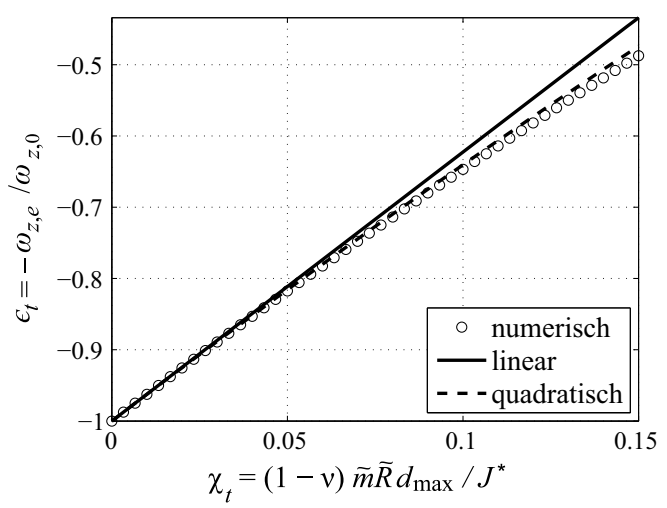

\subsubsection{Stoß mit Gleiten}

Nach der Lösung für den Fall der Abwesenheit lokalen Gleitens (also für einen sehr großen Reibbeiwert), soll nun der Einfluss lokalen Gleitens untersucht werden. Dazu wird zunächst die analytische Lösung für Parameterkombinationen gezeigt, in denen der Kontakt während des gesamten Stoßes vollständig gleitet. Obwohl diese Situation für den rein torsionalen Stoß nie tatsächlich realisierbar ist ${ }^{5}$, stellt diese Lösung, wie sich zeigen wird, in vielen Fällen eine ausgezeichnete Näherung dar.

\section{Lösung für vollständig gleitenden Kontakt}

Das Torsionsmoment für den vollständig gleitenden Kontakt ist wegen Gl. (3.150) durch

$$
M_{z}=-\frac{\mu \pi}{4} \tilde{E} \frac{a^{4}}{\tilde{R}}
$$

gegeben. Die Bewegungsgleichung kann daher mit den in Gl. (7.36) eingeführten normierten Größen in der Form

$$
\frac{\mathrm{d} \hat{\omega}_{z}}{\mathrm{~d} \hat{t}}+\frac{15 \mu \pi}{64} \frac{\tilde{m} \tilde{R}^{2}}{J^{*}}\left(\frac{d_{\max }}{\tilde{R}}\right)^{3 / 2} \xi^{4 / 5}=0
$$

geschrieben werden, die man während der Kompressionsphase mithilfe von Gl. (7.39) zu

$$
\hat{\omega}_{z}(\xi)=\hat{\omega}_{z, 0}-\frac{3 \mu \pi}{32} \frac{\tilde{m} \tilde{R}^{2}}{J^{*}}\left(\frac{d_{\max }}{\tilde{R}}\right)^{3 / 2} \mathrm{~B}\left(\xi ; \frac{6}{5}, \frac{1}{2}\right),
$$

mit der im Anhang beschriebenen Beta-Funktion B, integrieren kann. Während der Restitutionsphase wird sich die Winkelgeschwindigkeit bei fortbestehendem vollständigen Gleiten

\footnotetext{
${ }^{5}$ Im Torsionskontakt mit Reibung gibt es immer eine endlich kleine Umgebung des Kontaktmittelpunktes, die lokal haftet.
} 
um den gleichen Betrag verringern wie während der Kompression, sodass sich als torsionale Stoßzahl in diesem Fall der Ausdruck

$$
\epsilon_{t}=-1+\frac{3 \pi}{16} \frac{\sqrt{\pi} \Gamma(1,2)}{\Gamma(1,7)} \frac{\chi_{t}}{\psi_{t}}, \quad \psi_{t}:=(1-v) \frac{a_{\max }\left|\omega_{z, 0}\right|}{\mu\left|v_{z, 0}\right|},
$$

ergibt. Der Parameter $\chi_{t}$ wurde bereits in Gl.(7.36) definiert, $\psi_{t}$ ist das Analogon des aus dem ebenen Stoß mit Gleiten bekannten Parameters $\psi$ für den Torsionsstoß.

\section{Allgemeine Lösung für partielles Gleiten}

Die Lösung im allgemeinen Fall partiellen Gleitens kann man mithilfe der im dritten Kapitel geschilderten kontaktmechanischen Grundlagen, am einfachsten mit ihrer Deutung im Rahmen der MDR, numerisch bestimmen. Die torsionale Stoßzahl hängt nur von den beiden Parametern $\chi_{t}$ und $\psi_{t}$ ab und ist in Abb. 7.5 als Funktion von $\psi_{t}$ für verschiedene Werte von $\chi_{t}$ gezeigt. Die dünnen Linien bezeichnen jeweils die analytische Lösung für vollständiges Gleiten, die offensichtlich für Werte $\psi_{t}>1$ fast perfekt mit der tatsächlichen Lösung übereinstimmt. Insgesamt nimmt die Stoßzahl mit steigendem $\psi_{t}$ betragsmäßig grundsätzlich $\mathrm{zu}$, in dem relevanten Bereich kleiner Werte von $\chi_{t}$ wird der Energieverlust während des Stoßes damit mit steigendem $\psi_{t}$, also beispielsweise für kleinere Reibkoeffizienten, kleiner.

\subsection{Zusammenfassung}

Bei der Rückführung (vom Standpunkt der makroskopischen Dynamik) der räumlichen Kollision von Kugeln auf den Stoß einer Kugel auf einen ruhenden Halbraum im zweiten Kapitel wurden mehrere Effekte als vernachlässigbar klein behandelt, die entsprechend nur in räumlichen Kollisionen auftreten. Zwei dieser Effekte wurden in dem vergangenen Kapitel genauer untersucht, zum einen der Einfluss der Zentrifugalkraft durch die Rotation

\begin{abstract}
Abb. 7.5 Torsionale Stoßzahl $\epsilon_{t}$ als Funktion des Parameter $\psi_{t}$ für verschiedene Werte von $\chi_{t}$ - siehe Gl. (7.36) - für den elastischen Torsionsstoß einer Kugel mit Gleiten. Die dünnen Linien beschreiben jeweils die in Gl. (7.58) gegebene Lösung für vollständiges Gleiten
\end{abstract}

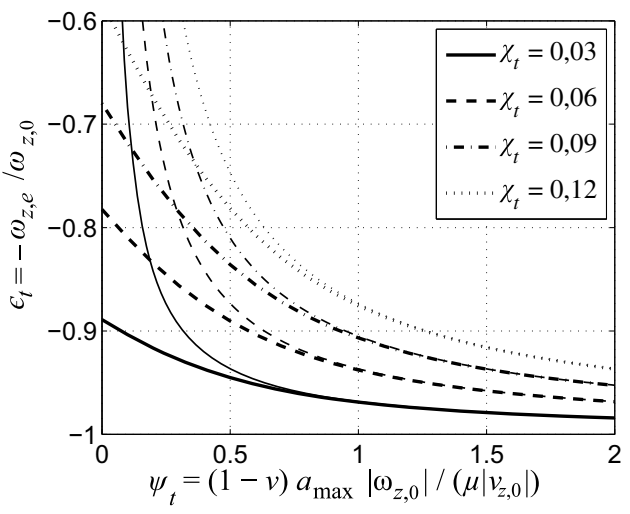


der Stoßachse während der Kollision und zum anderen die elastische Deformation durch die Torsion. Da die betrachteten Effekte klein sind (bzw. langsam ablaufen), können die auftretenden Gleichungen asymptotisch in geschlossener Form gelöst werden, auch wenn eine allgemeine analytische Behandlung unmöglich ist.

Die Zentrifugalkraft durch die Rotation der Stoßachse ist für besonders flache Stoßwinkel von Bedeutung und sorgt für eine zusätzliche Abstoßung der Kugeln während der Kollision. Dadurch sind die maximale Eindrucktiefe und die Stoßdauer kleiner als im Fall ohne zentrifugale Beiträge zur Normalkraft. Die zusätzliche Repulsion ist speziell für adhäsive Stöße interessant, da sich dadurch auch kollidierende Kugeln wieder voneinander lösen können, deren relative Normalgeschwindigkeit dafür in einem reinen Normalstoß zu klein wäre. In Stößen mit Reibung sorgt die zusätzliche Abstoßung dafür, dass die normale Stoßzahl (wenn sie kinematisch als Verhältnis von Geschwindigkeiten nach und vor dem Stoß definiert wird) auch in elastischen Kollisionen ohne Adhäsion kleiner als Eins ist.

Die torsionale Stoßzahl für den elastischen reinen Torsionsstoß mit Gleiten hängt, wie die tangentiale Stoßzahl im schiefen elastischen Stoß mit Gleiten, von zwei Parametern ab, von denen der erste die geometrischen und der zweite die Reibeigenschaften des Kontaktes charakterisiert. Der Energieverlust während des torsionalen Stoßes nimmt mit steigendem Reibkoeffizienten grundsätzlich zu.

\section{Literatur}

1. Jäger, J. (1994). Torsional impact of elastic spheres. Archive of Applied Mechanics, 64(4), 235248.

Open Access Dieses Kapitel wird unter der Creative Commons Namensnennung 4.0 International Lizenz (http://creativecommons.org/licenses/by/4.0/deed.de) veröffentlicht, welche die Nutzung, Vervielfältigung, Bearbeitung, Verbreitung und Wiedergabe in jeglichem Medium und Format erlaubt, sofern Sie den/die ursprünglichen Autor(en) und die Quelle ordnungsgemäß nennen, einen Link zur Creative Commons Lizenz beifügen und angeben, ob Änderungen vorgenommen wurden.

Die in diesem Kapitel enthaltenen Bilder und sonstiges Drittmaterial unterliegen ebenfalls der genannten Creative Commons Lizenz, sofern sich aus der Abbildungslegende nichts anderes ergibt. Sofern das betreffende Material nicht unter der genannten Creative Commons Lizenz steht und die betreffende Handlung nicht nach gesetzlichen Vorschriften erlaubt ist, ist für die oben aufgeführten Weiterverwendungen des Materials die Einwilligung des jeweiligen Rechteinhabers einzuholen.

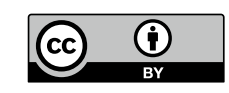

